\title{
Donor-Stabilized Cations and Imine Transfer from N-Silylphosphoranimines
}

\author{
Eric Rivard, Keith Huynh, Alan J. Lough and Ian Manners* \\ Department of Chemistry, Davenport Laboratories, University of Toronto \\ 80 St. George Street, Toronto, Ontario, Canada M5S 3H6
}

\section{Supporting Information}

(1) Experimental Section

(2) Table 1. Equilibrium between [3] Cl and free $\mathbf{1}$ and DMAP in the presence of added chloride ion

(3) Table 2. Crystal data and structure refinement for [3]OTf

(4) Table 3. Atomic coordinates and equivalent isotropic displacement parameters for [3]OTf

(5) Table 4. Bond lengths and angles for [3]OTf

(6) Table 5. Anisotropic parameters for [3]OTf

(7) Table 6. Hydrogen coordinates and isotropic displacement parameters for [3]OTf 


\section{Experimental Section}

All reactions and manipulations were carried out under an atmosphere of prepurified nitrogen or argon (Air Products) using common Schlenk techniques or an inert atmosphere glove box (M-Braun). Solvents were dried and collected using a Grubbs-type solvent purification system manufactured by M-Braun. ${ }^{1}{ }^{1} \mathrm{H}$ and ${ }^{31} \mathrm{P}\left\{{ }^{1} \mathrm{H}\right\}$ NMR spectra were obtained on a Varian Gemini 300 spectrometer $(300.1$ and $121.5 \mathrm{MHz}$ ) and were referenced either to protic impurities in the solvent $\left({ }^{1} \mathrm{H}\right)$ or externally to $85 \% \mathrm{H}_{3} \mathrm{PO}_{4}\left({ }^{31} \mathrm{P}\left\{{ }^{1} \mathrm{H}\right\}\right)$ in $\mathrm{CDCl}_{3}, \mathrm{CD}_{2} \mathrm{Cl}_{2}$ or $\mathrm{D}_{2} \mathrm{O}$ (insert). ${ }^{13} \mathrm{C}\left\{{ }^{1} \mathrm{H}\right\}$ and ${ }^{29} \mathrm{Si}\left\{{ }^{1} \mathrm{H}\right\}$ NMR spectra were obtained on a Varian Unity 400 spectrometer $(100.5$ and $79.4 \mathrm{MHz}$ ) and were both referenced externally to $\mathrm{SiMe}_{4}$ in $\mathrm{CDCl}_{3}$. Mass spectra were obtained with the use of a VG-250S mass spectrometer using a $70 \mathrm{eV}$ electron impact ionization source. Melting points (uncorrected) were obtained in $0.5 \mathrm{~mm}$ (o.d.) glass capillaries which were flame sealed under nitrogen. Elemental analyses were performed at the University of Toronto using a Perkin-Elmer 2400 Series CHN Analyzer. 4-Dimethylaminopyridine (DMAP) was obtained from Aldrich and used as received. The silver salts $\mathrm{Ag}[\mathrm{OTf}], \mathrm{Ag}\left[\mathrm{BF}_{4}\right]$ and $\mathrm{Ag}\left[\mathrm{SbF}_{6}\right]$ were also obtained from Aldrich and were dried under dynamic vacuum at $100{ }^{\circ} \mathrm{C}$ for $24 \mathrm{~h}$ prior to use. Triphenylphosphine (BDH Chemicals) and tetra-n-octylammonium bromide (Aldrich) were dried in vacuo for $24 \mathrm{~h}$ prior to use. $\left[\mathrm{Ph}_{3} \mathrm{P}=\mathrm{N}=\mathrm{PPh}_{3}\right] \mathrm{Cl}$ (Aldrich) was recrystallized from $\mathrm{CH}_{2} \mathrm{Cl}_{2}$ and dried in vacuo at $100{ }^{\circ} \mathrm{C}$ for $16 \mathrm{~h}$ before use. Tri-n-butylphosphine (Aldrich) was vacuum distilled and stored under an atmosphere of nitrogen. $\mathrm{Cl}_{3} \mathrm{P}=\mathrm{NSiMe}_{3}$ (1) was prepared according to a literature procedure. ${ }^{2}$

Xray Structure Determination of [3]OTf. Data were collected on a Nonius Kappa-CCD diffractometer using graphite-monochromated Mo K $\alpha$ radiation $(\lambda=0.71073 \AA)$. A combination of $1^{\circ} \phi$ and $\omega$ (with $\kappa$ offsets) scans were used to collect sufficient data. The data frames were integrated and scaled using the Denzo-SMN package. ${ }^{3}$ The structures were solved and refined with the SHELXTL-PC v6.12 software package. ${ }^{4}$ Refinement was by full-matrix least squares on $F^{2}$ using data (including negative intensities) with hydrogen atoms bonded to carbon atoms included in calculated positions and treated as riding atoms.

Preparation of [DMAP• $\mathbf{C l}_{2} \mathbf{P}=\mathbf{N S i M e}_{3} \mathbf{O S O}_{2} \mathbf{C F}_{3}$, [3] OTf. To a mixture of DMAP (1.06 g, 8.67 $\mathrm{mmol})$ and $\mathrm{Ag}\left[\mathrm{OSO}_{2} \mathrm{CF}_{3}\right](2.21 \mathrm{~g}, 8.63 \mathrm{mmol})$ in $20 \mathrm{~mL}$ of $\mathrm{CH}_{2} \mathrm{Cl}_{2}$ was added dropwise a $3 \mathrm{~mL}$ solution of $\mathrm{Cl}_{3} \mathrm{P}=\mathrm{NSiMe}_{3}(1.94 \mathrm{~g}, 8.63 \mathrm{mmol})$ in $\mathrm{CH}_{2} \mathrm{Cl}_{2}$ at $25^{\circ} \mathrm{C}$ (in the absence of light). A white precipitate formed immediately and the reaction was stirred for $1 \mathrm{~h}$. The reaction mixture was then filtered and slow evaporation of the filtrate under nitrogen $(16 \mathrm{~h})$ afforded large colorless needles of [3]OTf (3.26 g, 82\%). Attempts to generate a stable phosphoranimine cation by the treatment of 1 with $\mathrm{Ag}[\mathrm{OTf}]$ in the presence of pyridine, bipyridine or without base, led to the formation of poly(dichlorophosphazene) $\left[{ }^{31} \mathrm{P} \mathrm{NMR}\left(\mathrm{CDCl}_{3}\right): \delta=-18.0 \mathrm{ppm}\right.$ (s)] and $\mathrm{Me}_{3} \operatorname{SiOTf}\left[{ }^{1} \mathrm{H} \mathrm{NMR}\left(\mathrm{CDCl}_{3}\right): \delta=0.51 \mathrm{ppm}(\mathrm{s}) ;{ }^{19} \mathrm{~F} \mathrm{NMR}\left(\mathrm{CDCl}_{3}\right): \delta=-78.0 \mathrm{ppm}(\mathrm{s})\right]$.

Data for [3]OTf: ${ }^{31} \mathrm{P}\left\{{ }^{1} \mathrm{H}\right\}$ NMR $\left(\mathrm{CDCl}_{3}\right): \delta=-39.8 \mathrm{ppm}(\mathrm{s}) .{ }^{1} \mathrm{H}$ NMR $\left(\mathrm{CDCl}_{3}\right): \delta=0.30(\mathrm{~s}$, $\left.\mathrm{SiMe}_{3}, 9 \mathrm{H}\right), 3.51$ (s, $\left.\mathrm{NMe}_{2}, 6 \mathrm{H}\right), 7.31$ (dd, $J=2.7$ and $8.4 \mathrm{~Hz}$, ortho- $\left.\mathrm{ArH}, 2 \mathrm{H}\right)$ and $8.46 \mathrm{ppm}(\mathrm{dd}$, $J=7.4$ and $10.7 \mathrm{~Hz}$, meta-ArH, $2 \mathrm{H}) .{ }^{13} \mathrm{C}\left\{{ }^{1} \mathrm{H}\right\} \mathrm{NMR}\left(\mathrm{CDCl}_{3}\right): \delta=1.7\left(\mathrm{~d},{ }^{3} J_{\mathrm{CP}}=5.0 \mathrm{~Hz}, \mathrm{SiMe}_{3}\right)$, $41.5\left(\mathrm{~s}, \mathrm{NMe}_{2}\right), 108.9\left(\mathrm{~d},{ }^{2} J_{\mathrm{CP}}=8.0 \mathrm{~Hz}\right.$, ortho-C (DMAP)), 120.5 (q, $\left.{ }^{1} J_{\mathrm{CF}}=166.1 \mathrm{~Hz}, \mathrm{OTf}\right), 138.7$ $\left(\mathrm{d},{ }^{3} J_{\mathrm{CP}}=5.0 \mathrm{~Hz}\right.$, meta-C (DMAP) $)$ and $157.8 \mathrm{ppm}$ (s, para-C (DMAP)). ${ }^{19} \mathrm{~F}$ NMR $\left(\mathrm{CDCl}_{3}\right): \delta=$ $78.2 \mathrm{ppm}$ (s, OTf). ${ }^{29} \mathrm{Si}\left\{{ }^{1} \mathrm{H}\right\} \mathrm{NMR}\left(\mathrm{CDCl}_{3}\right): \delta=2.5 \mathrm{ppm}\left(\mathrm{d},{ }^{2} J_{\mathrm{SiP}}=11.9 \mathrm{~Hz}\right) . \mathrm{mp}\left({ }^{\circ} \mathrm{C}\right): 98-102$ (dec). EI-MS (70 eV, m/z, \%): $312\left(\mathrm{M}^{+}-\mathrm{OTf}, 2\right), 208\left(\mathrm{Cl}_{3} \mathrm{P}=\mathrm{NSiMe}_{3}-\mathrm{Me}, 1\right), 189$ $\left(\mathrm{Cl}_{2} \mathrm{P}=\mathrm{NSiMe}_{3}, 4\right), 155\left(\mathrm{ClP}=\mathrm{NSiMe}_{3}, 8\right), 121\left(\mathrm{DMAP}^{+}, 100\right), 69\left(\mathrm{CF}_{3}^{+}, 64\right)$. Anal. Calcd. for $\mathrm{C}_{11} \mathrm{H}_{19} \mathrm{Cl}_{2} \mathrm{~F}_{3} \mathrm{~N}_{3} \mathrm{O}_{3} \mathrm{PSSi}(460.3)$ : $\% \mathrm{C}: 28.70$; $\% \mathrm{H}: 4.16$; $\% \mathrm{~N}$ : 9.13. Found: $\% \mathrm{C}: 28.50 ; \% \mathrm{H}: 3.98$; $\% \mathrm{~N}: 9.05$. 
Preparation of [DMAP• $\mathbf{C l}_{2} \mathbf{P}=\mathbf{N S i M e}$ ] Cl, [3]Cl. To a solution of $\mathrm{Cl}_{3} \mathrm{P}=\mathrm{NSiMe}_{3}(93 \mathrm{mg}, 0.41$ mmol) in $1 \mathrm{~mL}$ of $\mathrm{CH}_{2} \mathrm{Cl}_{2}$ was added dropwise a $0.5 \mathrm{~mL}$ solution of DMAP (47 mg, $\left.0.38 \mathrm{mmol}\right)$ in $\mathrm{CH}_{2} \mathrm{Cl}_{2}$. The resulting colorless solution was stirred for $16 \mathrm{~h}$ and the volatiles (including any excess $\mathrm{Cl}_{3} \mathrm{P}=\mathrm{NSiMe}_{3}$ ) were removed in vacuo to give a white solid (110 mg, $\left.82 \%\right)$.

${ }^{31} \mathrm{P}\left\{{ }^{1} \mathrm{H}\right\}$ NMR $\left(\mathrm{CDCl}_{3}\right): \delta=-39.2 \mathrm{ppm}(\mathrm{s}) .{ }^{1} \mathrm{H}$ NMR $\left(\mathrm{CDCl}_{3}\right): \delta=0.08\left(\mathrm{~s}, \mathrm{SiMe}_{3}, 9 \mathrm{H}\right), 3.46(\mathrm{~s}$, $\left.\mathrm{NMe}_{2}, 6 \mathrm{H}\right), 7.65$ (dd, ortho-ArH, $2 \mathrm{H}, J=3.0$ and $\left.8.1 \mathrm{~Hz}\right)$ and $8.37 \mathrm{ppm}(\mathrm{dd}$, meta-ArH, $2 \mathrm{H}, J=$ 8.4 and $10.1 \mathrm{~Hz}) \cdot{ }^{13} \mathrm{C}\left\{{ }^{1} \mathrm{H}\right\} \mathrm{NMR}\left(\mathrm{CDCl}_{3}\right): \delta=1.7\left(\mathrm{~d},{ }^{3} J_{\mathrm{CP}}=4.5 \mathrm{~Hz}, \mathrm{SiMe}_{3}\right), 42.1\left(\mathrm{~s}, \mathrm{NMe}_{2}\right), 109.7$ $\left(\mathrm{d},{ }^{2} J_{\mathrm{CP}}=8.6 \mathrm{~Hz}\right.$, ortho-C (DMAP)), $138.6\left(\mathrm{~d},{ }^{3} J_{\mathrm{CP}}=5.9 \mathrm{~Hz}\right.$, meta-C (DMAP)) and $157.8 \mathrm{ppm}(\mathrm{s}$, para-C (DMAP)). ${ }^{29} \mathrm{Si}\left\{{ }^{1} \mathrm{H}\right\} \mathrm{NMR}\left(\mathrm{CDCl}_{3}\right): \delta=2.4 \mathrm{ppm}\left(\mathrm{d},{ }^{2} J_{\mathrm{SiP}}=11.1 \mathrm{~Hz}\right)$.

To investigate the equilibrium nature of [3]Cl, sequential amounts of chloride ion, as $\left[\mathrm{Ph}_{3} \mathrm{P}=\mathrm{N}=\mathrm{PPh}_{3}\right] \mathrm{Cl}$, was added to a freshly prepared solution of [3] Cl in $\mathrm{CDCl}_{3}$ (see Table 1). One equiv. of bromide ion, $\left[{ }^{\mathrm{n}} \mathrm{Oct}{ }_{4} \mathrm{~N}\right] \mathrm{Br}$, did not react with the $[3]^{+}$cation.

Preparation and Decomposition of $\left[\mathrm{DMAP} \cdot \mathrm{Cl}_{2} \mathbf{P}=\mathrm{NSiMe}_{3}\right] \mathbf{B F}_{4},[3] \mathbf{B F}_{4}$. In the absence of light, a solution of $\mathrm{Cl}_{3} \mathrm{P}=\mathrm{NSiMe}_{3}(0.28 \mathrm{~g}, 0.12 \mathrm{mmol})$ in $1 \mathrm{~mL}$ of dichloromethane was added to a mixture of DMAP $(0.15 \mathrm{~g}, 0.12 \mathrm{mmol})$ and $\mathrm{Ag}\left[\mathrm{BF}_{4}\right](0.25 \mathrm{~g}, 0.13 \mathrm{mmol})$ in $2 \mathrm{~mL}$ of $\mathrm{CH}_{2} \mathrm{Cl}_{2}$. The reaction was stirred for $1 \mathrm{~h}$ to give a white suspension. The $\mathrm{AgCl}$ was filtered off and the volatiles were removed from the filtrate to give a white solid $(0.31 \mathrm{~g}, 62 \%)$ which was identified as [3]BF 4 by NMR spectroscopy. ${ }^{31} \mathrm{P}\left\{{ }^{1} \mathrm{H}\right\}$ NMR $\left(\mathrm{CDCl}_{3}\right): \delta=-39.8(\mathrm{~s}) \mathrm{ppm} .{ }^{1} \mathrm{H}$ NMR $\left(\mathrm{CDCl}_{3}\right): \delta=$ 0.25 (s, 9H, $\left.\mathrm{SiMe}_{3}\right), 3.44$ (s, 6H, NMe $), 7.20$ (br, 2H, ortho ArH) and $8.40 \mathrm{ppm}$ (br, meta $\left.\mathrm{ArH}\right)$. ${ }^{19} \mathrm{~F} \mathrm{NMR}\left(\mathrm{CDCl}_{3}\right): \delta=-151.4 \mathrm{ppm}$ (pseudoquartet, $\left.\mathrm{BF}_{4}{ }^{-}\right)$.

Compound $[3] \mathbf{B F}_{4}$ gradually decomposed over the period of 3 days in $\mathrm{CDCl}_{3}$ to give $\left[\mathrm{Cl}_{2} \mathrm{P}=\mathrm{N}\right]_{3}$ and $\mathrm{DMAP} \cdot \mathrm{BF}_{3}$ (see below).

Data for decomposition products: ${ }^{31} \mathrm{P}\left\{{ }^{1} \mathrm{H}\right\} \mathrm{NMR}\left(\mathrm{CDCl}_{3}\right): \delta=20.0 \mathrm{ppm}\left(\mathrm{s},\left[\mathrm{Cl}_{2} \mathrm{P}=\mathrm{N}\right]_{3}\right) .{ }^{1} \mathrm{H}$ NMR $\left(\mathrm{CDCl}_{3}\right): \delta=3.20\left(\mathrm{~s}, 6 \mathrm{H}, \mathrm{NMe}_{2}\right), 6.66(\mathrm{~d}, 2 \mathrm{H}$, ortho $\mathrm{ArH}, J=7.0 \mathrm{~Hz})$ and $8.1 \mathrm{ppm}$ (br, meta $\mathrm{ArH}) .{ }^{19} \mathrm{~F}$ NMR $\left(\mathrm{CDCl}_{3}\right): \delta=-151.6 \mathrm{ppm}(\mathrm{m}) .{ }^{11} \mathrm{~B}$ NMR $\left(\mathrm{CDCl}_{3}\right): \delta=-0.02$ (br). Lit. for

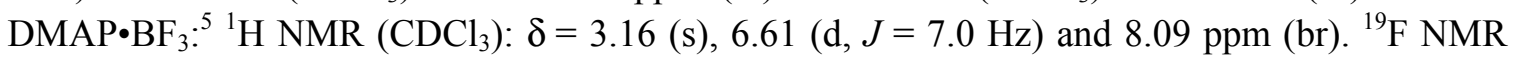
$\left(\mathrm{CDCl}_{3}\right): \delta=-152.7 \mathrm{ppm}(\mathrm{m}) .{ }^{11} \mathrm{~B} \mathrm{NMR}\left(\mathrm{CDCl}_{3}\right): \delta=0.34 \mathrm{ppm}(\mathrm{br})$.

In Situ Preparation of $\left[\mathbf{D M A P} \cdot \mathbf{C l}_{2} \mathbf{P}=\mathrm{NSiMe}_{3}\right] \mathbf{S b F}_{6},[3] \mathbf{S b F}_{6}$. In the absence of light, a $0.5 \mathrm{~mL}$ solution of 1 (90 mg, $0.40 \mathrm{mmol}$ ) in $\mathrm{CDCl}_{3}$ was added quickly to a stirred mixture of $\mathrm{Ag}\left[\mathrm{SbF}_{6}\right]$ $(138 \mathrm{mg}, 0.40 \mathrm{mmol})$ and DMAP (49 $\mathrm{mg}, 0.40 \mathrm{mmol})$ in $1 \mathrm{~mL}$ of $\mathrm{CDCl}_{3}$. The resulting grey suspension was stirred for $1 \mathrm{~h}$ and the $\mathrm{AgCl}$ was filtered off. Analysis of the resulting colorless filtrate by NMR indicated the formation of $[3] \mathbf{S b F}_{\mathbf{6}}\left(95 \%\right.$ pure; trace of unreacted $\mathbf{1},{ }^{31} \mathrm{P}:-54.0$ ppm (s), was present). Compound [3] $\mathbf{S b} \mathbf{F}_{\mathbf{6}}$ was stable in solution for up to two weeks without any noticeable sign of decomposition.

${ }^{31} \mathrm{P}\left\{{ }^{1} \mathrm{H}\right\} \mathrm{NMR}\left(\mathrm{CDCl}_{3}\right): \delta=-40.2 \mathrm{ppm}(\mathrm{s}) .{ }^{1} \mathrm{H} \mathrm{NMR}\left(\mathrm{CDCl}_{3}\right): \delta=0.24\left(\mathrm{~s}, 9 \mathrm{H}, \mathrm{SiMe}_{3}\right), 3.38(\mathrm{~s}$, $\left.6 \mathrm{H}, \mathrm{NMe}_{2}\right), 7.04(\mathrm{dd}, 2 \mathrm{H}$, ortho $\mathrm{ArH}, J=3.2$ and $7.1 \mathrm{~Hz})$ and $8.34 \mathrm{ppm}(\mathrm{dd}$, meta $\mathrm{ArH}, J=8.1$ and $11.1 \mathrm{~Hz}) .{ }^{19} \mathrm{~F} \mathrm{NMR}\left(\mathrm{CDCl}_{3}\right): \delta=-110$ (very broad) and $-135 \mathrm{ppm}$ (very broad) $\left(\mathrm{SbF}_{6}{ }^{-}\right)$.

Preparation of $\mathbf{P h}_{3} \mathbf{P}=\mathbf{N}-\mathbf{P C l}_{\mathbf{2}}$ (7) from $\mathbf{P h}_{3} \mathbf{P}$ and $\mathbf{C l}_{3} \mathbf{P}=\mathbf{N S i M e}$. A solution of $\mathrm{Ph}_{3} \mathrm{P}(0.34 \mathrm{~g}, 1.3$ $\mathrm{mmol})$ in $3 \mathrm{~mL}$ of $\mathrm{CH}_{2} \mathrm{Cl}_{2}$ was added to a solution of $1(0.29 \mathrm{~g}, 1.3 \mathrm{mmol})$ in $2 \mathrm{~mL}$ of $\mathrm{CH}_{2} \mathrm{Cl}_{2}$. The reaction was stirred for $3 \mathrm{~h}$ and removal of the volatiles afforded a white solid which was characterized as the previously known phosphoranimine $\mathrm{Ph}_{3} \mathrm{P}=\mathrm{N}-\mathrm{PCl}_{2}(7)^{6}(0.43 \mathrm{~g}, 89 \%)$. Repeating the reaction in $\mathrm{CDCl}_{3}$ also identified $\mathrm{ClSiMe}_{3}$ as a byproduct $\left[{ }^{1} \mathrm{H}\right.$ NMR: $\delta=0.45 \mathrm{ppm}$ (s)]. 
Data for 7: ${ }^{31} \mathrm{P}$ NMR $\left(\mathrm{CDCl}_{3}\right): \delta=165.6\left(\mathrm{~d},{ }^{2} J_{\mathrm{PP}}=75.0 \mathrm{~Hz},-\mathrm{PCl}_{2}\right)$ and $15.8 \mathrm{ppm}\left(\mathrm{d},{ }^{2} J_{\mathrm{PP}}=75.0\right.$ $\left.\mathrm{Hz}, \mathrm{Ph}_{3} P=\mathrm{N}-\right) .{ }^{1} \mathrm{H} \mathrm{NMR}\left(\mathrm{CDCl}_{3}\right): \delta=7.5-8.0 \mathrm{ppm}(\mathrm{m})$.

In Situ Preparation of ${ }^{\mathrm{n}} \mathrm{Bu}_{3} \mathrm{P}=\mathbf{N}-\mathrm{PCl}_{2}(8)$ from ${ }^{\mathrm{n}} \mathrm{Bu}_{3} \mathbf{P}$ and $\mathrm{Cl}_{3} \mathrm{P}=\mathrm{NSiMe}_{3}$. To a solution of $\mathbf{1}$ $(0.52 \mathrm{~g}, 2.3 \mathrm{mmol})$ in $5 \mathrm{~mL}$ of $\mathrm{CH}_{2} \mathrm{Cl}_{2}$ was added ${ }^{\mathrm{n}} \mathrm{Bu}_{3} \mathrm{P}(0.60 \mathrm{~mL}, 2.4 \mathrm{mmol})$ dropwise. Analysis of the resulting yellow solution after $2 \mathrm{~h}$ revealed the formation of $\mathrm{PCl}_{3}(\delta=218.0 \mathrm{ppm}$ (s); ca. 5 $\%),{ }^{\mathrm{n}} \mathrm{Bu}_{3} \mathrm{P}=\mathrm{NSiMe}_{3}(\delta=-8.8 \mathrm{ppm}(\mathrm{s}) ;$ ca. $5 \%),{ }^{\mathrm{n}} \mathrm{Bu}_{3} \mathrm{P}=\mathrm{N}-\mathrm{PCl}_{2}(\mathbf{8})\left[\delta=158.0\left(\mathrm{~d},{ }^{2} J_{\mathrm{PP}}=85.0 \mathrm{~Hz},-\right.\right.$ $\left.P \mathrm{Pl}_{2}\right)$ and $40.7 \mathrm{ppm}\left(\mathrm{d},{ }^{2} J_{\mathrm{PP}}=85.0 \mathrm{~Hz},{ }^{\mathrm{n}} \mathrm{Bu}_{3} P=\mathrm{N}-\right)$; $1: 1 \mathrm{ratio} ; c a .55 \%$ ] and an unidentified species at $\delta=105.1$ (s, ca. $25 \%$ ) and $35.1 \mathrm{ppm}$ (s, ca. $10 \%$ ). Stirring the reaction for further 6 days gave an $80 \%$ (in situ) yield of ${ }^{\mathrm{n}} \mathrm{Bu}_{3} \mathrm{P}=\mathrm{N}-\mathrm{PCl}_{2}{ }^{7}$ and unknown species (ca. $20 \%$ ) with ${ }^{31} \mathrm{P}$ NMR resonances at $\delta=72(\mathrm{br})$ and $35 \mathrm{ppm}$ (s). Attempts to isolate pure 8 have yet to be successful.

\section{References:}

(1) Pangborn, A. B.; Giardello, M. A.; Grubbs, R. H.; Rosen, R. K.; Timmers, F. J. Organometallics 1996, 15, 1518.

(2) Wang, B.; Rivard, E.; Manners, I. Inorg. Chem. 2002, 41, 1690.

(3) Otwinowski, Z.; Minor, W. Methods Enzymol. 1997, 276, 367.

(4) Sheldrick, G. M. SHELXTL-Windows NT. V6.12, Bruker Analytical X-Ray Systems Inc., Madison, WI, 2001.

(5) Lesley, M. J. G.; Woodward, A.; Taylor, N. J.; Marder, T. M.; Cazenobe, I.; Ledoux, I.; Zyss, J.; Thornton, A.; Bruce, D. W.; Kakkar, A. K. Chem. Mater. 1998, 10, 1355.

(6) Riesel, L.; Friebe, R. Z. Anorg. Allg. Chem. 1981, 474, 105

(7) Riesel, L.; Friebe, R. Z. Anorg. Allg. Chem. 1991, 604, 85. 
Table 1 Equilibrium between [3]Cl and free $\mathbf{1}$ and DMAP in the presence of added chloride ion $^{\mathrm{a}}$

\begin{tabular}{cccc}
\hline $\begin{array}{c}\text { Molar equiv. } \\
\mathrm{Cl}_{3} \mathrm{P}=\mathrm{NSiMe}_{3}{ }^{\mathrm{a}} \\
\text { of chloride }^{\mathrm{c}}\end{array}$ & Mol \% of $[\mathbf{3}] \mathbf{C l}^{\mathrm{b}}$ & Mol & of \\
\hline 0 & 95 & 5 \\
0.25 & 80 & 20 \\
0.5 & 60 & 40 \\
0.75 & 25 & 75 \\
1.0 & 0 & 100
\end{tabular}

${ }^{\mathrm{a}}$ As determined by ${ }^{31} \mathrm{P}$ NMR spectroscopy. ${ }^{\mathrm{b}}$ Initial concentration of [3]Cl $(0.3-0.4 \mathrm{M})$; reaction time, 1.5 h. $^{\mathrm{c}}$ Source of chloride: $\left[\mathrm{Ph}_{3} \mathrm{P}=\mathrm{N}=\mathrm{PPh}_{3}\right] \mathrm{Cl}$. 
Table 2. Crystal data and structure refinement for [3]OTf.

Identification code

Empirical formula

Formula weight

Temperature

Wavelength

Crystal system

Space group

Unit cell dimensions

Volume

Z

Density (calculated)

Absorption coefficient

$\mathrm{F}(000)$

Crystal size

Theta range for data collection

Index ranges

Reflections collected

Independent reflections

Completeness to theta $=27.54^{\circ}$

Absorption correction

Max. and min. transmission

Refinement method

Data / restraints / parameters

Goodness-of-fit on $\mathrm{F}^{2}$

Final $\mathrm{R}$ indices [I $>2 \operatorname{sigma}(\mathrm{I})]$

$\mathrm{R}$ indices (all data)

Extinction coefficient

Largest diff. peak and hole k0384

C11 H19 Cl2 F3 N3 O3 P S Si

460.31

150(1) K

$0.71073 \AA$

Triclinic

P-1

$\mathrm{a}=8.0840(4) \AA$

$\alpha=88.5630(19)^{\circ}$.

$\mathrm{b}=8.6430(4) \AA$

$\beta=86.5950(18)^{\circ}$.

$\mathrm{c}=14.6080(7) \AA$

$\gamma=85.038(2)^{\circ}$.

2

$1.506 \mathrm{Mg} / \mathrm{m}^{3}$

$0.602 \mathrm{~mm}^{-1}$

472

$0.24 \times 0.16 \times 0.08 \mathrm{~mm}^{3}$

2.77 to $27.54^{\circ}$.

$-10<=\mathrm{h}<=10,-11<=\mathrm{k}<=11,-18<=\mathrm{l}<=18$

13104

$4659[\mathrm{R}(\mathrm{int})=0.0986]$

$99.5 \%$

Semi-empirical from equivalents

0.970 and 0.783

Full-matrix least-squares on $\mathrm{F}^{2}$

4659 / 0 / 232

0.987

$\mathrm{R} 1=0.0550, \mathrm{wR} 2=0.1243$

$\mathrm{R} 1=0.1090, \mathrm{wR} 2=0.1513$

$0.005(2)$

0.451 and -0.535 e. $\AA^{-3}$ 
Table 3. Atomic coordinates ( $\left.\mathrm{x} 10^{4}\right)$ and equivalent isotropic displacement parameters $\left(\AA^{2} \times 10^{3}\right)$ for [3]OTf. U(eq) is defined as one third of the trace of the orthogonalized $U^{i j}$ tensor.

\begin{tabular}{|c|c|c|c|c|}
\hline & $\mathrm{x}$ & $\mathrm{y}$ & z & $\mathrm{U}(\mathrm{eq})$ \\
\hline $\mathrm{Cl}(1)$ & $2350(1)$ & $3626(1)$ & $6608(1)$ & $37(1)$ \\
\hline $\mathrm{Cl}(2)$ & $5498(1)$ & $4335(1)$ & $7610(1)$ & $38(1)$ \\
\hline $\mathrm{P}(1)$ & $4565(1)$ & 2644(1) & $6939(1)$ & $28(1)$ \\
\hline $\operatorname{Si}(1)$ & $6815(1)$ & 2056(1) & $5196(1)$ & $29(1)$ \\
\hline $\mathrm{N}(1)$ & 4045(3) & 1394(3) & $7818(2)$ & $25(1)$ \\
\hline $\mathrm{N}(2)$ & 3097(3) & $-1681(3)$ & $9967(2)$ & $27(1)$ \\
\hline $\mathrm{N}(3)$ & $5660(4)$ & 1814(4) & $6219(2)$ & $32(1)$ \\
\hline$C(1)$ & $3033(4)$ & $1869(4)$ & $8581(2)$ & $29(1)$ \\
\hline$C(2)$ & 2718(4) & $895(4)$ & $9285(2)$ & $27(1)$ \\
\hline$C(3)$ & 3394(4) & $-679(4)$ & $9288(2)$ & $24(1)$ \\
\hline$C(4)$ & 4441(4) & $-1141(4)$ & $8492(2)$ & $31(1)$ \\
\hline$C(5)$ & 4741(4) & $-121(4)$ & $7808(2)$ & $31(1)$ \\
\hline$C(7)$ & 1982(5) & $-1292(5)$ & $10774(2)$ & $35(1)$ \\
\hline $\mathrm{C}(8)$ & $3730(5)$ & $-3335(4)$ & 9891(3) & $33(1)$ \\
\hline $\mathrm{C}(9)$ & $7726(5)$ & $112(4)$ & 4841(3) & $39(1)$ \\
\hline$C(10)$ & $5420(5)$ & $2895(5)$ & $4316(3)$ & $40(1)$ \\
\hline $\mathrm{C}(11)$ & $8467(5)$ & $3337(5)$ & $5392(3)$ & $40(1)$ \\
\hline$S(1)$ & $9386(1)$ & $-3591(1)$ & $8633(1)$ & $30(1)$ \\
\hline $\mathrm{F}(1)$ & 8649(3) & $-1395(3)$ & $7411(2)$ & $62(1)$ \\
\hline $\mathrm{F}(2)$ & $11106(3)$ & $-2527(3)$ & $7206(2)$ & $65(1)$ \\
\hline $\mathrm{F}(3)$ & $9035(4)$ & $-3691(3)$ & $6860(2)$ & $69(1)$ \\
\hline $\mathrm{O}(1)$ & $10414(4)$ & $-5033(3)$ & $8570(2)$ & $52(1)$ \\
\hline $\mathrm{O}(2)$ & 10004(3) & $-2411(3)$ & $9165(2)$ & $42(1)$ \\
\hline $\mathrm{O}(3)$ & $7630(3)$ & $-3736(3)$ & $8780(2)$ & $41(1)$ \\
\hline$C(12)$ & 9543(5) & $-2770(5)$ & $7473(3)$ & $41(1)$ \\
\hline
\end{tabular}


Table 4. Bond lengths $[\AA]$ and angles $\left[^{\circ}\right]$ for [3]OTf.

\begin{tabular}{|c|c|}
\hline $\mathrm{Cl}(1)-\mathrm{P}(1)$ & $1.9947(12)$ \\
\hline $\mathrm{Cl}(2)-\mathrm{P}(1)$ & $2.0040(13)$ \\
\hline $\mathrm{P}(1)-\mathrm{N}(3)$ & $1.490(3)$ \\
\hline $\mathrm{P}(1)-\mathrm{N}(1)$ & $1.713(3)$ \\
\hline $\mathrm{Si}(1)-\mathrm{N}(3)$ & $1.733(3)$ \\
\hline $\operatorname{Si}(1)-C(11)$ & $1.846(4)$ \\
\hline $\mathrm{Si}(1)-\mathrm{C}(9)$ & $1.849(4)$ \\
\hline $\operatorname{Si}(1)-C(10)$ & $1.854(4)$ \\
\hline $\mathrm{N}(1)-\mathrm{C}(5)$ & $1.380(4)$ \\
\hline $\mathrm{N}(1)-\mathrm{C}(1)$ & $1.392(4)$ \\
\hline $\mathrm{N}(2)-\mathrm{C}(3)$ & $1.325(4)$ \\
\hline $\mathrm{N}(2)-\mathrm{C}(7)$ & $1.468(4)$ \\
\hline $\mathrm{N}(2)-\mathrm{C}(8)$ & $1.480(4)$ \\
\hline$C(1)-C(2)$ & $1.339(5)$ \\
\hline $\mathrm{C}(2)-\mathrm{C}(3)$ & $1.422(5)$ \\
\hline$C(3)-C(4)$ & $1.440(5)$ \\
\hline$C(4)-C(5)$ & $1.341(5)$ \\
\hline $\mathrm{S}(1)-\mathrm{O}(2)$ & $1.438(3)$ \\
\hline $\mathrm{S}(1)-\mathrm{O}(1)$ & $1.439(3)$ \\
\hline $\mathrm{S}(1)-\mathrm{O}(3)$ & $1.439(3)$ \\
\hline$S(1)-C(12)$ & $1.822(4)$ \\
\hline $\mathrm{F}(1)-\mathrm{C}(12)$ & $1.341(4)$ \\
\hline $\mathrm{F}(2)-\mathrm{C}(12)$ & $1.333(4)$ \\
\hline $\mathrm{F}(3)-\mathrm{C}(12)$ & $1.318(5)$ \\
\hline $\mathrm{N}(3)-\mathrm{P}(1)-\mathrm{N}(1)$ & $110.73(15)$ \\
\hline $\mathrm{N}(3)-\mathrm{P}(1)-\mathrm{Cl}(1)$ & 118.92(13) \\
\hline $\mathrm{N}(1)-\mathrm{P}(1)-\mathrm{Cl}(1)$ & $102.36(10)$ \\
\hline $\mathrm{N}(3)-\mathrm{P}(1)-\mathrm{Cl}(2)$ & $117.90(13)$ \\
\hline $\mathrm{N}(1)-\mathrm{P}(1)-\mathrm{Cl}(2)$ & $101.91(11)$ \\
\hline $\mathrm{Cl}(1)-\mathrm{P}(1)-\mathrm{Cl}(2)$ & $102.68(6)$ \\
\hline $\mathrm{N}(3)-\mathrm{Si}(1)-\mathrm{C}(11)$ & $108.60(17)$ \\
\hline $\mathrm{N}(3)-\mathrm{Si}(1)-\mathrm{C}(9)$ & 107.37(17) \\
\hline $\mathrm{C}(11)-\mathrm{Si}(1)-\mathrm{C}(9)$ & $110.65(18)$ \\
\hline
\end{tabular}




\begin{tabular}{|c|c|}
\hline $\mathrm{N}(3)-\mathrm{Si}(1)-\mathrm{C}(10)$ & $109.16(16)$ \\
\hline$C(11)-\operatorname{Si}(1)-C(10)$ & $111.36(18)$ \\
\hline C(9)-Si(1)-C(10) & 109.60(19) \\
\hline$C(5)-N(1)-C(1)$ & $117.9(3)$ \\
\hline C(5)-N(1)-P(1) & 119.3(2) \\
\hline $\mathrm{C}(1)-\mathrm{N}(1)-\mathrm{P}(1)$ & $122.6(2)$ \\
\hline $\mathrm{C}(3)-\mathrm{N}(2)-\mathrm{C}(7)$ & $123.3(3)$ \\
\hline $\mathrm{C}(3)-\mathrm{N}(2)-\mathrm{C}(8)$ & $120.5(3)$ \\
\hline $\mathrm{C}(7)-\mathrm{N}(2)-\mathrm{C}(8)$ & $115.8(3)$ \\
\hline $\mathrm{P}(1)-\mathrm{N}(3)-\mathrm{Si}(1)$ & $144.1(2)$ \\
\hline $\mathrm{C}(2)-\mathrm{C}(1)-\mathrm{N}(1)$ & $121.7(3)$ \\
\hline $\mathrm{C}(1)-\mathrm{C}(2)-\mathrm{C}(3)$ & $121.6(3)$ \\
\hline $\mathrm{N}(2)-\mathrm{C}(3)-\mathrm{C}(2)$ & $123.4(3)$ \\
\hline $\mathrm{N}(2)-\mathrm{C}(3)-\mathrm{C}(4)$ & $121.0(3)$ \\
\hline $\mathrm{C}(2)-\mathrm{C}(3)-\mathrm{C}(4)$ & $115.6(3)$ \\
\hline$C(5)-C(4)-C(3)$ & $120.8(3)$ \\
\hline $\mathrm{C}(4)-\mathrm{C}(5)-\mathrm{N}(1)$ & $122.3(3)$ \\
\hline $\mathrm{O}(2)-\mathrm{S}(1)-\mathrm{O}(1)$ & $115.75(18)$ \\
\hline $\mathrm{O}(2)-\mathrm{S}(1)-\mathrm{O}(3)$ & $114.50(16)$ \\
\hline $\mathrm{O}(1)-\mathrm{S}(1)-\mathrm{O}(3)$ & $115.36(18)$ \\
\hline $\mathrm{O}(2)-\mathrm{S}(1)-\mathrm{C}(12)$ & $102.37(19)$ \\
\hline $\mathrm{O}(1)-\mathrm{S}(1)-\mathrm{C}(12)$ & $103.64(17)$ \\
\hline $\mathrm{O}(3)-\mathrm{S}(1)-\mathrm{C}(12)$ & $102.51(17)$ \\
\hline $\mathrm{F}(3)-\mathrm{C}(12)-\mathrm{F}(2)$ & $106.1(3)$ \\
\hline $\mathrm{F}(3)-\mathrm{C}(12)-\mathrm{F}(1)$ & $108.0(3)$ \\
\hline $\mathrm{F}(2)-\mathrm{C}(12)-\mathrm{F}(1)$ & $106.7(3)$ \\
\hline $\mathrm{F}(3)-\mathrm{C}(12)-\mathrm{S}(1)$ & $112.5(3)$ \\
\hline $\mathrm{F}(2)-\mathrm{C}(12)-\mathrm{S}(1)$ & 111.8(3) \\
\hline $\mathrm{F}(1)-\mathrm{C}(12)-\mathrm{S}(1)$ & $111.4(3)$ \\
\hline
\end{tabular}

Symmetry transformations used to generate equivalent atoms: 
Table 5. Anisotropic displacement parameters $\left(\AA^{2} \times 10^{3}\right)$ for [3]OTf. The anisotropic displacement factor exponent takes the form: $-2 \pi^{2}\left[h^{2} a^{* 2} U^{11}+\ldots+2 h \mathrm{k}^{*} b^{*} U^{12}\right]$

\begin{tabular}{|c|c|c|c|c|c|c|}
\hline & $\mathrm{U}^{11}$ & $\mathrm{U}^{22}$ & $\mathrm{U}^{33}$ & $\mathrm{U}^{23}$ & $\mathrm{U}^{13}$ & $\mathrm{U}^{12}$ \\
\hline $\mathrm{Cl}(1)$ & $32(1)$ & $40(1)$ & $38(1)$ & $3(1)$ & $-4(1)$ & $6(1)$ \\
\hline $\mathrm{Cl}(2)$ & $49(1)$ & $35(1)$ & $33(1)$ & $3(1)$ & $-6(1)$ & $-14(1)$ \\
\hline $\mathrm{P}(1)$ & $28(1)$ & $28(1)$ & $26(1)$ & $3(1)$ & $0(1)$ & $-3(1)$ \\
\hline $\operatorname{Si}(1)$ & $26(1)$ & $32(1)$ & $28(1)$ & $0(1)$ & $3(1)$ & $-4(1)$ \\
\hline $\mathrm{N}(1)$ & $29(2)$ & $22(1)$ & $25(2)$ & $-4(1)$ & $3(1)$ & $0(1)$ \\
\hline $\mathrm{N}(2)$ & $29(2)$ & $25(2)$ & $27(2)$ & $0(1)$ & $1(1)$ & 1(1) \\
\hline $\mathrm{N}(3)$ & $30(2)$ & $35(2)$ & $30(2)$ & $3(1)$ & $4(1)$ & $-2(1)$ \\
\hline $\mathrm{C}(1)$ & $25(2)$ & $28(2)$ & $32(2)$ & $1(2)$ & $3(1)$ & $1(2)$ \\
\hline$C(2)$ & $27(2)$ & $25(2)$ & $28(2)$ & $-3(2)$ & $5(1)$ & $-2(2)$ \\
\hline$C(3)$ & $22(2)$ & $26(2)$ & $24(2)$ & $0(1)$ & $-1(1)$ & $-3(1)$ \\
\hline$C(4)$ & $29(2)$ & $24(2)$ & $36(2)$ & $0(2)$ & $5(2)$ & $4(2)$ \\
\hline$C(5)$ & $31(2)$ & $27(2)$ & $32(2)$ & $-2(2)$ & $6(2)$ & $1(2)$ \\
\hline$C(7)$ & $34(2)$ & $42(2)$ & $29(2)$ & $6(2)$ & $3(2)$ & $-3(2)$ \\
\hline$C(8)$ & $39(2)$ & $23(2)$ & $37(2)$ & $3(2)$ & $-1(2)$ & $0(2)$ \\
\hline$C(9)$ & $35(2)$ & $37(2)$ & $46(2)$ & $-6(2)$ & $6(2)$ & $-4(2)$ \\
\hline$C(10)$ & $41(2)$ & $46(2)$ & $32(2)$ & $7(2)$ & $2(2)$ & $-9(2)$ \\
\hline $\mathrm{C}(11)$ & $35(2)$ & $45(2)$ & $41(2)$ & $-4(2)$ & $0(2)$ & $-13(2)$ \\
\hline $\mathrm{S}(1)$ & $28(1)$ & $31(1)$ & $29(1)$ & $-2(1)$ & $3(1)$ & $2(1)$ \\
\hline $\mathrm{F}(1)$ & $71(2)$ & $57(2)$ & $51(2)$ & $21(1)$ & 11(1) & $18(1)$ \\
\hline $\mathrm{F}(2)$ & $46(2)$ & $82(2)$ & $61(2)$ & $14(2)$ & $27(1)$ & $-5(1)$ \\
\hline $\mathrm{F}(3)$ & $88(2)$ & $85(2)$ & $34(1)$ & $-12(1)$ & $-2(1)$ & $-14(2)$ \\
\hline $\mathrm{O}(1)$ & $59(2)$ & $40(2)$ & $51(2)$ & $-1(1)$ & $6(1)$ & $25(2)$ \\
\hline $\mathrm{O}(2)$ & $39(2)$ & $49(2)$ & $40(2)$ & $-12(1)$ & $-3(1)$ & $-9(1)$ \\
\hline $\mathrm{O}(3)$ & $29(1)$ & $49(2)$ & $45(2)$ & $5(1)$ & $6(1)$ & $-8(1)$ \\
\hline $\mathrm{C}(12)$ & $37(2)$ & $47(2)$ & $36(2)$ & $1(2)$ & $8(2)$ & $6(2)$ \\
\hline
\end{tabular}


Table 6. Hydrogen coordinates ( $\left.\times 10^{4}\right)$ and isotropic displacement parameters $\left(\AA^{2} \times 10^{3}\right)$ for $\mathrm{k} 0384$.

\begin{tabular}{|c|c|c|c|c|}
\hline & $\mathrm{x}$ & $\mathrm{y}$ & $\mathrm{z}$ & $\mathrm{U}(\mathrm{eq})$ \\
\hline $\mathrm{H}(1 \mathrm{~A})$ & 2552 & 2911 & 8603 & 34 \\
\hline $\mathrm{H}(2 \mathrm{~A})$ & 2027 & 1266 & 9796 & 32 \\
\hline $\mathrm{H}(4 \mathrm{~A})$ & 4924 & -2180 & 8452 & 37 \\
\hline $\mathrm{H}(5 \mathrm{~A})$ & 5459 & -453 & 7299 & 37 \\
\hline $\mathrm{H}(7 \mathrm{~A})$ & 1478 & -226 & 10705 & 53 \\
\hline $\mathrm{H}(7 \mathrm{~B})$ & 2619 & -1380 & 11326 & 53 \\
\hline $\mathrm{H}(7 \mathrm{C})$ & 1106 & -2011 & 10828 & 53 \\
\hline $\mathrm{H}(8 \mathrm{~A})$ & 4946 & -3414 & 9807 & 50 \\
\hline $\mathrm{H}(8 \mathrm{~B})$ & 3262 & -3784 & 9365 & 50 \\
\hline $\mathrm{H}(8 \mathrm{C})$ & 3400 & -3902 & 10453 & 50 \\
\hline $\mathrm{H}(9 \mathrm{~A})$ & 8376 & -372 & 5334 & 59 \\
\hline $\mathrm{H}(9 \mathrm{~B})$ & 8453 & 215 & 4285 & 59 \\
\hline $\mathrm{H}(9 \mathrm{C})$ & 6833 & -537 & 4716 & 59 \\
\hline $\mathrm{H}(10 \mathrm{~A})$ & 4903 & 3907 & 4517 & 59 \\
\hline $\mathrm{H}(10 \mathrm{~B})$ & 4553 & 2195 & 4230 & 59 \\
\hline $\mathrm{H}(10 \mathrm{C})$ & 6066 & 3028 & 3734 & 59 \\
\hline $\mathrm{H}(11 \mathrm{~A})$ & 9154 & 2885 & 5878 & 60 \\
\hline $\mathrm{H}(11 \mathrm{~B})$ & 7961 & 4362 & 5577 & 60 \\
\hline $\mathrm{H}(11 \mathrm{C})$ & 9163 & 3445 & 4825 & 60 \\
\hline
\end{tabular}

